\title{
Utilization Review of Imaging Equipment: An insight into CT Scanning
}

\author{
Vijay Kumar Tadia ${ }^{1}$, Shakti Kumar Gupta ${ }^{2}$, Sidhartha Satpathy ${ }^{3}$, \\ Arun Kumar Gupta ${ }^{4}$, Sanjay Kumar Arya ${ }^{5}$ \\ ${ }^{1}$ Senior Resident Administrator, Department of Hospital Administration' AIIMS, New Delhi, ${ }^{2}$ Medical \\ Superintendent, Dr. R. P. Centre for Ophthalmic Sciences, AIIMS, New Delhi, ${ }^{3}$ Professor and Head, Department \\ of Hospital Administration, AIIMS, New Delhi, ${ }^{4}$ Professor and Head, Department of Radiodiagnosis, AIIMS, New \\ Delhi, ${ }^{5}$ Professor, Department of Hospital Administration, AIIMS, New Delhi
}

\begin{abstract}
Introduction: Radio diagnosis service plays a crucial role in diagnosis of the patient's disease. The volume of patients utilising this service is very high. This service also takes away the sizeable share of total capital investment and therefore, the optimal utilization of this service becomes important.
\end{abstract}

Methodology: Literature review was done through Google scholar, electronic database of PubMed and other relevant databases. The query terms like Utilization Review of Imaging Equipment, Utilisation of CT Scanners, Capacity of Scanners in Radiology and Waiting Times for Imaging were used to fetch the relevant data sources. Around 130 articles were reviewed and analysed.

Results: The number of CT Scans per 1 million of population in High Income (HI), Upper Middle Income (UMI), Lower Middle Income (LMI) are 14.7, 7.3, 3.7. The CT scanners are one of the most expensive pieces of medical equipment in hospitals, with CT scanners costing between $\$ 1$ million (₹7 crore) and \$2 million (₹14 crore).

There is a substantial variation in the utilisation of these services, including number of scans per day and wait times. Increasing the scanner efficiency and utilisationcan significantly decrease the cost per scan and increase access to imaging services.

Expanding capacity, improving operating procedures by reducing change-over times, and improving capacity allocation in combination with scheduling procedures can contribute to the overall reduction of CT access time.

Discussion and Conclusion: By acquiring Utilization data about CT Scanners, we get to know a complete and accurate overview of how Scanners are used. The CT scanner utilisation can be improved in both public and private health sector by analysingthe key information about the utilisation of their own and other health services' CT scanners.

Keywords: Imaging Utilization, CT Scanner Efficiency, Radiology Productivity.

\section{Introduction}

Modern radiology departments and imaging centres are complex organizations that require costly equipment,

\section{Corresponding Author:}

Vijay Kumar Tadia

Senior Resident Administrator, Department of Hospital

Administration, AIIMS, New Delhi-110049

e-mail: vijaytadia@gmail.com sophisticated information systems, and a diversity of human resources to accomplish their missions. Managing these enterprises is challenging and achieving high levels of productivity and efficiency can be elusive. Healthcare organizations, including departments of radiology, are turning to industrial models for establishingrobust operational systems. Radio diagnosis service plays a crucial role in diagnosis of the patient's disease. The volume of patients utilising this service is very high. This service also takes away the sizeable share of total 
capital investment and therefore, the optimal utilization of this service becomes important.

\section{Methodology}

Literature review was done through Google scholar, electronicdatabase of PubMed and other relevant databases. The query terms like Utilization Review of Imaging Equipment, Utilisation of CT Scanners, Capacity of Scanners in Radiology and Waiting Times for Imaging were used to fetch the relevant data sources. Around 130 articles were reviewed and analysed.

\section{Results}

The number of CT Scans per 1 million of population in High Income (HI), Upper Middle Income (UMI), Lower Middle Income (LMI) are 14.7, 7.3, 3.7. In India, the number of CT Scanners available per million population is $3^{1}$. There were about 3000 scanners throughout India in year $2008^{2}$. Internationally, rates of CT exams per 1,000 range from highs of 275 (Estonia), 265 (United States) and 126 (Canada) to lows of 50 (Chile) and 66 (Netherlands) ${ }^{3}$.

Although conspicuous consumption is common, when it comes to CT scanners, quality is vastly superior to quantity. The goal of a successful CT installation should be to acquire more scans, not more scanners, through proper workflow management, according to a presentation at the International Symposium on Multidetector-Row CT, sponsored by Stanford University of Stanford, $\mathrm{CA}^{4}$.

The importance of productivity with expensive imaging equipment has been brought to the forefront of public discussion due to rising cost of healthcare. In particular, the use of imaging modalities such as magnetic resonance (MR) imaging, computed tomography $(\mathrm{CT})$, and positron emission tomography (PET) have been considered as contributing to the rising costs of healthcare ${ }^{5}$.

Public healthcare routinely use computed tomography (CT) scanners to diagnose, manage and treat many conditions. These scanners take high-quality images of internal organs and tissues. They are critical to clinical decisions in a patient's treatment, and can significantly influence patient outcomes. CT scanners are one of the most expensive pieces of medical equipment in hospitals, with CT scanners costing between \$1 million (₹7 crore) and \$2 million (₹14 crore). These equipment represent a considerable financial risk for hospitals due to their short life cycle of seven to 10 years, excluding major upgrades, and their high replacement and maintenance costs - up to $\$ 180000$ or more annually ${ }^{6}$.

The Victorian Auditor-General's Report 2014-15 revealed that the cost-effectiveness of delivering $\mathrm{CT}$ and MR imaging services varies widely across health services. Some CT and MR imaging services operate at a surplus while others incur losses each year. It further added that public health services were not managing CT and MR scanners efficiently or cost effectively. There was a substantial variation in the utilisation of these services, including number of scans per day and wait times. Hospitals were not able to compare the efficiency and economy of their scanners. Without the data that would enable this comparison it was difficult for health services and the Department of Health and Human Services to know whether costly imaging equipment was being used efficiently. The high capital and maintenance costs of computed tomography (CT) and magnetic resonance (MR) scanners mean that they should be used to their full capacity. Increasing the scanner efficiency and utilisationcan significantly decrease the cost per scan and increase access to imaging services. The managers of health services do not know whether costly imaging equipment is being utilised efficiently, which makes it difficult to take appropriate action to increase the efficiency. The findings of the report also revealed that there was a widespread variation in the utilisation of CT and MR imaging equipment-from 351 to over 21000 scans per machine per year; public health services did not systematically collect the information necessary to determine or improve the efficiency of the scanners; public health services had no means to compare their efficiency with that of other health services. The auditor analysed data from 315297 CT scans across 15 health services in 2012-13. There was wide variation in the total number of scans and operating hours of CT machines located within and outside EDs. Scanner productivity also varied in the number of scans performed per hour and the estimated downtime of scanners in operating hours. Scanner efficiency varied from less than one CT scan every two hours to more than three an hour, with an average of 2.3 scans per hour. Higher performing CT scanners completed eight times as many scans per hour as lower performing scanners. Thus, there is a significant opportunity to increase the efficiency of public CT scanners ${ }^{6}$.

The quantum of sophisticated technology and cost 
ofdiagnostic imaging, such as CT and MRI, has increased substantially over the years. At the same time, hospitals are forced to contain their costs and consequently do not extend their capacity at the same rate. Without taking steps for utilization management, the access (waiting) times shall remain prolonged ${ }^{7}$.Increasing the productivity is a pragmatic option to maintain costs, but this may conflict with achieving acceptable access times. Expanding capacity, improving operating procedures by reducing change-over times, and improving capacity allocation in combination with scheduling procedures can contribute to the overall reduction of CT access time. Capacity expansion is a rather expensive solution ${ }^{8}$. Improved operating procedures may create more CT capacity.

Dr. Sanjay Saini, Director of CT services at Massachusetts General Hospital (MGH) and a Professor of Radiology at Harvard Medical School, both in Boston had commented at the presentation made at the International Symposium on Multidetector-Row CT, sponsored by Stanford University of Stanford, CA.that the key was to focus on room turnaround time. He had further added that theoretically, one patient can be scanned every 15 minutes and it takes 20 seconds to do the examination and 14 1/2 minutes to do everything else and typically, a CT scanner does about 10,000 exams (annually $)^{4}$.

Boland et al (2006) report that some emergency department CT scanners perform more than 20,000 examinations annually9. The Victorian AuditorGeneral's Report 2014-15 also mentioneed that utilisation of CT imaging equipment can go as high as 21,000 scans per machine per year ${ }^{6}$.

Boland et al (2008) $)^{8}$ in a study entitled, "Maximizing Outpatient Computed Tomography Productivity Using Multiple Technologists" pointed out that Patients can be scheduled at shorter time intervals, preferably every 10 to 15 minutes with the 2-technologist model or every 10 minutes with the 3-technologist model.

At $1^{\text {st }}$ Biograph World Summit, Munich, Germany, Paul Shreve of Spectrum Health Grand Rapids (Grand Rapids, MI USA) recommended a time slot of 15 minutes between CT Scans for Optimized CT Utilization ${ }^{10}$.

In a study by Van Lent WAM et al at the Netherlands Cancer Institute - Antoni van Leeuwenhoek Hospital (NKI-AVL), a comprehensive cancer centre, located in The Netherlands, the available capacity of One CT
Scanner was divided in assigned places in the schedule of $10 \mathrm{~min}$ (also called slots). The slots were filled with $\mathrm{CT}$ requests, once a request was performed they called it an examination. An examination usually consumed one slot, but one examination may sometimes be composed of multiple procedures that may require multiple slots. The CT access time decreased from 9.8 to 7.3 days; this effect was caused by the new capacity allocation. They used Monte Carlo Simulation Model to see the effects of proposed scenarios to change the capacity allocated to different requests. Simulation was selected as a preferred OR technique as it is commonly applied in healthcare. They collected the data from Radiology Information System (RIS). They commented that this was first study of its kind reporting on operations research based change in Radiology that presented results of pre and post implementation analysis. By increasing the number of slots, the capacity allocation was optimized. The modelled outcomes included a growth of $6 \%$ in slots, the post implementation analysis showed a $1 \%$ growth in slots and $14 \%$ growth in examinations. Besides the tangible effects, the model increased the awareness that optimizing capacity allocation can reduce access times $^{7,11}$.

A prospective study for a period of six months carried out at Sher-i-Kashmir Institute of Medical Sciences, Srinagar, India for the calculation of unit cost of radiological investigations of CT head, CT chest, CT abdomen found out that the actual cost incurred by the hospital on CT head was Rupees 581.40 (US \$10.89), CT abdomen Rupees 2339.20 (US \$43.83), CT chest Rupees 2339.20 (US \$43.83). Out of this, the material cost of CT film, film covers and other stationary items was $16.9 \%$ of total cost in case of CT Head and $15.6 \%$ of total cost in case of CT Chest/CT Abdomen ${ }^{12}$. This makes a case for effective utilization of CT Scanners.

According to American Board of Radiology,all imaging departments are expected to establish and maintain effective quality, safety, and performance improvement programs. Essential components of such programs include adherence to the basic principles of quality management and appropriate utilization of quality tools. Quality improvement efforts can facilitate continuous improvement in safety, performance, and outcomes in the radiology department. Many of these efforts are now mandated by regulatory organizations. For example, radiologists must actively participate in a Practice Quality Improvement project approved by the American Board of Radiology to meet ongoing 
Maintenance of Certification requirements. In radiology, the focus of quality improvement is to improve the performance of and processes related to diagnostic and therapeutic procedures, the selection of imaging and procedural services, the quality and safety of healthcare delivered, and the effectiveness and management of all imaging services. ${ }^{13}$

The Imaging Center Subcommittee of the Radiology Business Management Association (RBMA) conducted a survey of its members and collected information on imaging center characteristics and equipment utilization by major imaging machine/modality (with data collected for each of the elements of the Medicare formula used to calculate equipment cost per minute) ${ }^{14}$. Web-based and Excel-based surveys were utilized because of the ease with which respondents could share it with others and print it for their own records. An invitation to participate in the survey with a link to the questionnaire was e-mailed on April 20th, 2009 to 1,084 RBMA members who have previously indicated they have imaging centers; this list included duplicate members from some practices.

Table 1. Results of Equipment Utilization Rate (RBMA)

\begin{tabular}{|l|l|c|}
\hline \multirow{4}{*}{$\begin{array}{l}\text { Availability-based Utilization } \\
\text { Rate }\end{array}$} & $\mathrm{N}$ & $\mathrm{CT}$ \\
\cline { 2 - 3 } & $\mathrm{Q} 1$ & 30 \\
\cline { 2 - 3 } & Mean & $31 \%$ \\
\cline { 2 - 3 } & Median & $62 \%$ \\
\cline { 2 - 3 } & $\mathrm{Q} 3$ & $59 \%$ \\
\hline \multirow{4}{*}{$\begin{array}{l}\text { Medicare-based Utilization } \\
\text { Rate }\end{array}$} & $\mathrm{N}$ & $90 \%$ \\
\cline { 2 - 3 } & Q1 & 29 \\
\cline { 2 - 3 } & Mean & $30 \%$ \\
\cline { 2 - 3 } & Median & $56 \%$ \\
\cline { 2 - 3 } & Q3 & $79 \%$ \\
\hline \multirow{4}{*}{$\begin{array}{l}\text { Uedicare-modified } \\
\text { Utization Rate }\end{array}$} & $\mathrm{N}$ & 25 \\
\cline { 2 - 3 } & Q1 & $39 \%$ \\
\cline { 2 - 3 } & Mean & $57 \%$ \\
\cline { 2 - 3 } & Median & $63 \%$ \\
\cline { 2 - 3 } & Q3 & $75 \%$ \\
\hline
\end{tabular}

In a study by Katherine P. Andriole, "Productivity and Cost Assessment of Computed Radiography, Digital Radiography, and Screen-Film for Outpatient Chest Examinations", the overall speed of service was calculated from the time of examination ordering as stamped in the radiology information system (RIS), to the time of image availability on the picture archiving and communication system (PACS), to the time of interpretation rendered (from the RIS). It was considered an objective assessment of computed radiography (CR) for performing upright chest examinations on outpatients is presented in terms of workflow, productivity, speed of service, and potential cost justification ${ }^{15}$.

In Quality Initiatives: Key Performance Indicators for Measuring and Improving Radiology Department Performance ${ }^{16}$, Hani H. Abujudeh, MD et al describe radiology-specific Key performance indicators (KPIs) that may help provide a framework for measuring performance in radiology practice. In healthcare organizations, performance assessment is especially critical for the development of best practices that can lead to improved outcomes in patient care, and KPIs have been incorporated into many healthcare management systems. Under operations management, it describes Utilization as one of the Strategic Areas of Radiology Department Performance. According to the Joint Commission on the Accreditation of Healthcare Organizations (JCAHO), a KPI is "a measurement tool used to monitor and evaluate the quality of important governance, management, clinical, and support functions" 17

A World Wide Web-based productivity tool called the Imaging Exam Time Monitor was developed to accurately and remotely monitor imaging efficiency with use of Digital Imaging and Communications in Medicine $(\mathrm{DICOM})^{18}$ combined with a picture archiving and communication system. Five device efficiency metricsexamination duration, table utilization, interpatient time, appointment interval time, and interseriestime-were derived from DICOM values. These metrics allow the standardized measurement of productivity, to facilitate the comparative evaluation of imaging equipment use and ongoing efforts to improve efficiency ${ }^{19}$. 


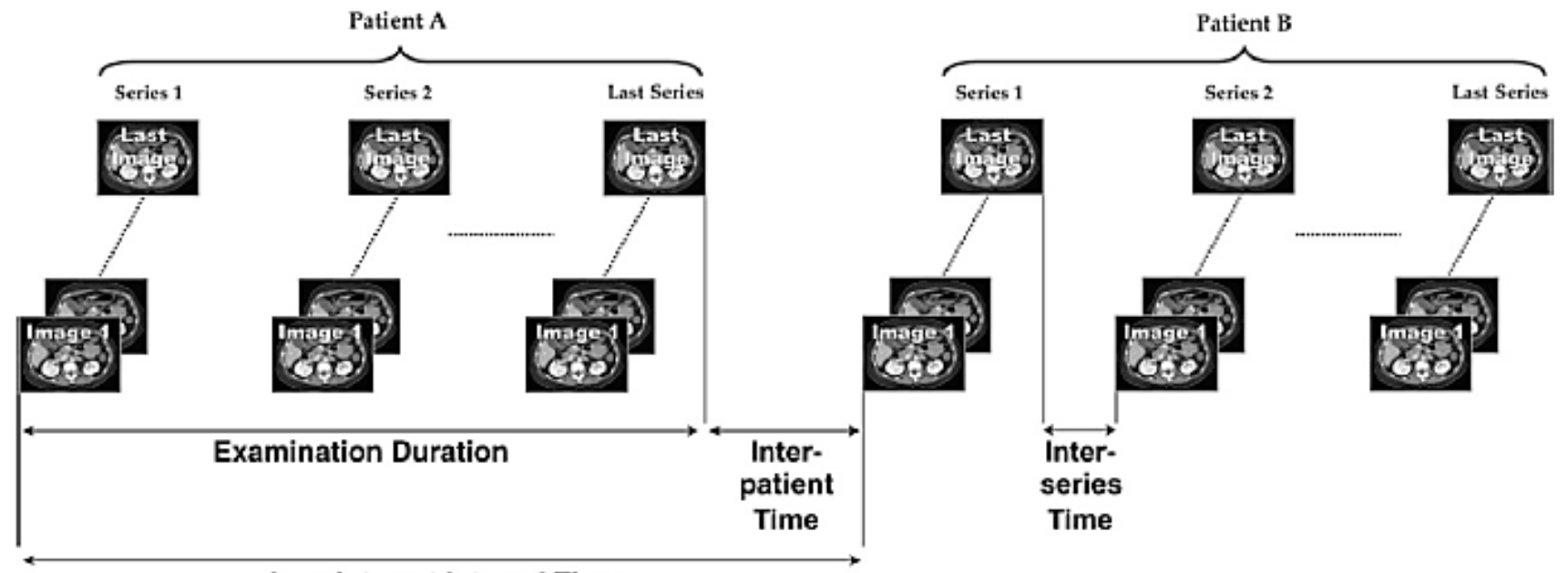

Appointment Interval Time

Source: Informatics in Radiology Efficiency Metrics for Imaging Device Productivity ${ }^{20}$

Figure 1. Metrics for examination duration time stamps as provided by DICOM.

Mengqi Hu in a study entitled, "A Study on Medical Imaging Equipment Productivity and Utilization" ${ }^{21}$ used DICOM Index Tracker to obtain accurate examination information in real time. In addition, they used Imaging Exam Time Monitor, a web based productivity tool is developed to accurately and transparently monitor imaging scanner efficiency for all devices and patients. They focussed on one of the productivity metrics called Examination Duration Time (EDT). The Examination Duration Time (EDT) has been defined as the time period during which a patient is being interrogated by an imaging device as recorded by PACS. It would be the time duration representative of the very first image on the first series to the time representative of last series last acquired image time as obtained from PACS. They conclude that such metrics allow us to continuously and automatically analyze the productivity data for all imaging equipment (e.g., CT, MG, MRI, et al.) which can be located in different departments, and hospitals, or even internationally in a comprehensive manner.

Like scientific data, quality data can be analyzed, and with a variety of similar tools ${ }^{22}$. Flow charts (discussed earlier), cause-and-effect diagrams, Pareto diagrams, check sheets, control charts, histograms, and scatter diagrams are commonly referred to as the seven basic tools of quality ${ }^{13}$.

The National Maximum Wait Time Access Targets for Medical Imaging (CT and MRI) have been given by Canadian Association of Radiologists (CAR), a member of the Wait Time Alliance (WTA) ${ }^{23}$. Since 2005, the
WTA has produced annual reports on the progress of addressing wait times in the five priority areas identified for focus in the 2004 First Ministers' Health Accord. One of those five priority areas was diagnostic imaging, particularly around MRI and CT.

The 2005 Benchmarks established by the CAR for the Wait Time Alliance (WTA) were as follows:

- Emergency cases - Immediate to $24 \mathrm{~h}$

- Urgent cases - Within 7 days

- Scheduled cases - Within 30 days.

Priority or urgency levels are defined as follows:

- Emergency $=$ Immediate danger to life, $\operatorname{limb}$ or organ

- $\quad$ Urgent $=$ Situation that is unstable and has the potential to deteriorate quickly and result in an emergency admission

- Scheduled $=$ Situation involving minimal pain, dysfunction or disability

\section{Discussion and Conclusion}

To capitalize fully on the investment made on state-of-the-art technology it is desirable to minimize the wasted time. By acquiring Utilization data about CT Scanners, we get to know a complete and accurate overview of how Scanners are used.

A defined set of nonarbitrary efficiency metrics are available that can be used and fully standardized across 
facilities. When coupled with other critical metrics developed from RIS-based utilization data such as interpretative report accuracy, completion time, number and types of patients examined, patient flow tracking systems, and so on, these standards provide a more complete picture of overall service productivity.

The key performance indicators for measuring and improving Radiology Department ${ }^{16}$ as given by Massachusetts General Hospital and Harvard Medical School, recommend the use of Imaging Exam Time Monitor, a web-based productivity tool to accurately and transparently monitor CT Scanner efficiency.

Boland et al $(2008)^{8}$ have demonstrated that parallel processing of tasks by two and three technologists during CT Scanning process can significantly reduce the total time to perform an examination. Their study shows that many tasks could be performed simultaneously by using additional personnel. Boland et al evaluated how many additional patients can be scanned using a 2- or 3-technologist model with outpatient multidetector CT and its impact on CT capacity. Their study concluded that CT room time per patient for 1-, 2-, and 3-technologist models was 12, 9.7, and 8.0 minutes, respectively. The number of patients scanned per hour for 1-, 2-, and 3-technologist models was 2.2, 5.2, and 7.5 , respectively. There was an increase of more than 12,000 potential patient CT slots made available using 2 technologists 7 days per week and 22,000 additional slots for a 3-technologist model when compared with a single-technologist model.

Similarly, Kutz et al (2005) $)^{24}$ applied Queuing Model to see the impact of extra technical assistant. They compared both a day and a week elapse based on ED CT data for 3 months and found that in case of one day lapse it led to $27 \%$ decrease in longest length of time a patient waits and $73 \%$ had CT within 30 minutes of being ordered. For one week lapse there was 33\% decrease in longest length of time a patient waits and $53 \%$ patients were having CT's in 18 minutes from being ordered. The new tech assistant was used to transport patient to CT Scanner.

At Newton-Wellesley Hospital, radiology turnaround stood at 45 minutes. After examining patient and technician flow, the hospital found that technicians spent too much time walking around. By redesigning the work flow, turnaround times fell to 25 minutes, making the planned addition of another $\$ 500,000$ ( $₹ 3.3$ crore) $\mathrm{x}$-ray machine unnecessary ${ }^{25}$.

Massachusetts General Hospital's proton beam facility was fully booked, or at least so the hospital thought until it did a little analysis. By batching patients requiring anaesthesia on the same day and scheduling an anaesthesiologist for that day, throughput increased from 29 patients per day to 39 patients - a 33 percent increase $^{25}$.

Intermountain Health Care (IHC) in Salt Lake City, Utah underwent a project to apply lean techniques to their processes. The project addressed a variety of issues and made a wide range of improvements. The processes that were improved significantly reduced amounts of wasted time by the workers. The reduction of hours required by employees can be translated into real savings almost immediately. Other savings could include reduced numbers of errors or increased patient satisfaction. Some examples of savings or benefits from the project included decreases in treatment delays and an immediate savings of nearly \$1 million (₹7 crore) through the implementation of an electronic payment system $^{26}$.

\section{Recommendations:}

1. It is recommended that different device efficiency metrics should be used for the Utilization Management of Radiology Equipment. DICOM tags (time stamps, patient exam type, device, etc) can be used to create customized and automatically generated Efficiency Reports. The Self-Generated Utilization Reports can save lot of tax payers money as subscription based services are chargeable.

2. Taking a clue from the Victorian Auditor-Generals Report 2014-15, a data repository to understand and compare the $\mathrm{CT}$ scanner utilisation in public health sector can be developed so that the public health services analyse and use key information about the utilisation of their own and other health services' CT scanners to maximise utilisation ${ }^{6}$.

3. More technologist(s) can be employed for each CT Scanner to increase the efficiency of CT Scanners further. Dr. Sanjay Saini, Director of CT services at Massachusetts General Hospital (MGH) and a Professor of radiology at Harvard Medical School, both in Boston has laid out some basic steps to keep a CT department running smoothly at maximum capacity". One of which is, "Idle technicians, not scanners". A good CT workflow strategy should be 
technologist-centered. Downtime for technologists is preferable to downtime for expensive machines. In the long run, overstaffing costs can be offset by having more people to do more exams and generating more income.

4. The Utilization Management Report for CT scanner provides with a large amount of useful data to help optimize system usage. The Utilization Management Report for CT helps to improve the usability and efficiency of Scanners. Utilization Management Services by Vendors like Siemens, Philips etc can be utilized to make best use of CT Scanners. Alternatively, in-house Utilization Management Dashboard can be developedfor all CT systems. Utilization Management must be an inbuilt feature in all the tenders/orders.

5. As detailed data on imaging equipment utilization is already collected remotely and electronically by equipment manufactures, it is recommended to follow suugestion made by Radiology Business Management Association (RBMA) ${ }^{14}$ to work with imaging providers, equipment manufacturers and other stakeholders, leveraging the use of existing information technology systems, to more accurately measure actual equipment utilization rates across a large and geographically diverse number of imaging centres.

6. A futuristic recommendation would be to set up National Wait Time Alliance based on Canadian Wait Time Alliance ${ }^{27}$. The National Maximum Wait Time Targets can be set for Medical Imaging (CT Scanning) similar to model of Canadian Association of Radiologists (CAR), a member of the Wait Time Alliance (WTA).

7. The DICOM time stamps do not in themselves fully describe the time the patient spends on the imaging table. But they record highly accurately the consistent measurements of exactly when patient imaging acquisition actually occurs in the context of a specific device. These times can be calibrated to the enterprise clock and the medical record, which can be coupled with other enterprise data systems.

8. It is suggested that different device efficiency metrics should be used for the Utilization Management of Radiology Equipment. DICOM tags (time stamps, patient exam type, device, etc) can be used to create customized and automatically generated Efficiency Reports. The Self-Generated Utilization Reports can save lot of tax payers money as subscription based services come at a premium from Vendors like Siemens, GE and Philips.

New Develpoments: The workflow of the existing scanning procedure depends heavily on the experience and subjective decisions of the technician, which often results in suboptimal image quality, large inter-technician variability, unnecessary radiation to the patient in case of CT, and prolonged scanning time. Therefore, Singh et $\mathrm{al}^{28}$ have proposed to equip the scanner with visual capability and the knowledge about the patient surface geometry to improve scanning in all these aspects. In order to provide the knowledge about the 3D patient surface, they have used a novel framework to obtain a detailed body surface model of the patient (on the table) using a range imaging device, which can ease scan planning in several ways. The estimated surface model includes a detailed body surface mesh as well as the location of various anatomical landmarks (such as the shoulders, thyroid, etc.) in the coordinate reference frame of the scanner. These surface landmarks provide a rough estimate of the organ positions which enables automatic table height adjustment. Use of such a technology can help to increase the efficiency of CT Scanners further. ${ }^{28}$

\section{Conflict of Interest: Nil}

\section{Source (s) of Support: Nil}

\section{Ethical Clearance: Not Required}

\section{References}

1. Singh H. Healthcare in India [Internet]. 2013 [cited 2016 Aug 27]. Available from: http://www.fujitsu. com/downloads/JP/archive/imgjp/group/fri/events/ other/20131024HarpalSigh.pdf

2. Jankharia G. Commentary - Radiology in India: The Next Decade. Indian J Radiol Imaging [Internet]. 2008 [cited 2016 Aug 27];18(3). Available from: http://www.ijri.org/temp/ IndianJRadiolImaging 183189-4459323_122313. pdf

3. Medical Imaging in Canada 2012 [Internet]. [cited 2016 Aug 27]. Available from: https://www.cihi. ca/en/mit_summary_2012_en.pdf

4. Pal S. Making the most of CT technology through efficient workflow [Internet]. 2004 [cited 2016 Aug 15]. Available from: http://www.auntminnie. com/index.aspx ?sec $=$ ser $\&$ sub=def \& pag=dis \& ItemID $=62165$ 
5. McGlynn EA, Shekelle PG, Goldman D, Romley J, Hussey P, de Vries H, etal. Identifying, Categorizing, and Evaluating Health Care Efficiency Measures [Internet]. 2008 [cited 2016 Aug 19]. Available from: http://archive.ahrq.gov/research/findings/ final-reports/efficiency/efficiency.pdf

6. Auditor-General V, Auditor-Generals Office V. Efficiency and Effectiveness of Hospital Services: High-value Equipment. 2015 [cited 2016 Sep 2]; Available from: http://www.audit. vic.gov.au/publications/20150225-HospitalEquipment/20150225-Hospital-Equipment.pdf

7. Van Lent WAM, Deetman JW, Teertstra HJ, Muller SH, Hans EW, Van Harten WH. Reducing the throughput time of the diagnostic track involving CT scanning with computer simulation. Eur J Radiol. 2012;81(11):3131-40.

8. Boland GWL, Houghton MP, Marchione DG, McCormick W. Maximizing Outpatient Computed Tomography Productivity Using Multiple Technologists. J Am Coll Radiol. 2008;5(2):11925.

9. Boland GWL. Stakeholder expectations for radiologists: Obstacles or opportunities? J Am Coll Radiol. 2006;3(3):156-63.

10. Shreve P. Optimized CT Utilization in PET CT. In: Molecular Imaging [Internet]. Grand Rapids, MI, USA: Siemens Healthcare; [cited 2016 Aug 22]. Available from: https://www.healthcare.siemens. com/molecular-imaging/customer-portal-resource/ customers-recordings

11. van Lent WAM. Improving resource capacity planning in hospitals with business approaches. 2011.

12. Rehana K, Tabish SA, Gojwari T, Ahmad R, Abdul H. Unit cost of CT scan and MRI at a large tertiary care teaching hospital in North India. 2013;5(12):2059-63.

13. Kruskal JB, Eisenberg R, Sosna J, Yam CS, Kruskal JD, Boiselle PM. Quality Initiatives: Quality Improvement in Radiology: Basic Principles and Tools Required to Achieve Success. Radiographics. 2011;31:1499-509.

14. Statistical Analyses by Sage Computing. Imaging Equipment Utilization Rates. 2009.

15. Andriole KP. Productivity and cost assessment of computed radiography, digital radiography, and screen-film for outpatient chest examinations. J
Digit Imaging. 2002;15(3):161-9.

16. Abujudeh HH, Kaewlai R, Asfaw BA, @bullet M, Thrall JH. QUALITY INITIATIVES: Key Performance Indicators for Measuring and Improving Radiology Department Performance.

17. Joint Commission on Accreditation of Healthcare Organizations. Primer on indicator development and application. 1990.

18. Digital Imaging and Communications in Medicine (DICOM) [Internet]. Vol. 3, PS. [cited 2016 Aug 19]. Available from: http://dicom.nema.org/ dicom/2001/01_01pu.pdf

19. Mengqi H, Pavlick W, Liu PT, Zhang M, Langer SG, Wang S, et al. Efficiency metrics for imaging device productivity. Radiogr J Contin Med Educ Radiol. 2011;31(2):603-16.

20. Utilization I, Law MYY, Liu B. Informatics in Radiology. Image Process [Internet]. 2009;29(3):655-68. Available from: http://www. ncbi.nlm.nih.gov/pubmed/19270073

21. Hu M. A Study on Medical Imaging Equipment Productivity and Utilization. In: Proceedings of the 2011 Industrial Engineering Research Conference. 2011. p. 1-9.

22. Tague N. The Quality Toolbox. Milwaukee, Wisconsin: American Society for Quality, Quality Press; 2005.

23. Canadian Association of Radiologists. National Maximum Wait Time Access Targets for Medical Imaging. 2013;(January). Available from: http:// www.car.ca/uploads/standards guidelines/car national_maximum_waittime_targets_mri_and ct_2013_en.pdf

24. Kutz T, Wilkerson J. Improvement of Emergency Department Operational Efficiency Through Streamlined CT Scanner Utilization. In ASQ, The Global Voice of Quality; 2015. Available from: https://asq.org/conferences/service-quality/2015/ program/sessions/session-t08.html

25. Arthur J. A Faster Hospital in Five Days [Internet]. Lean Six Sigma Demystified, Second Edition. McGraw Hill Professional, Access Engineering; 2011. Available from: https:// accessengineeringlibrary.com:443/browse/ lean-six-sigma-demystified-second-edition/ c9780071749091ch03

26. Jimmerson C, Missoula C, Weber D, Sobek DK. Reducing Waste and Errors: Piloting Lean 
Principles at IHC.

27. Wait Time Alliance Canadian Healthcare / Canadian Wait Times | Temps d'attente du Canada [Internet]. Available from: http://www.waittimealliance.ca/
28. Singh V, Chang YJ, Ma K, Wels M, Soza G, Chen T. Estimating a patient surface model for optimizing the medical scanning workflow. Lect Notes Comput Sci (including Subser Lect Notes Artif Intell Lect Notes Bioinformatics). 2014;8673 LNCS(PART 1):472-9. 